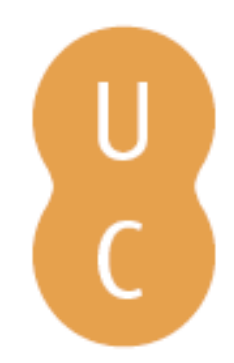

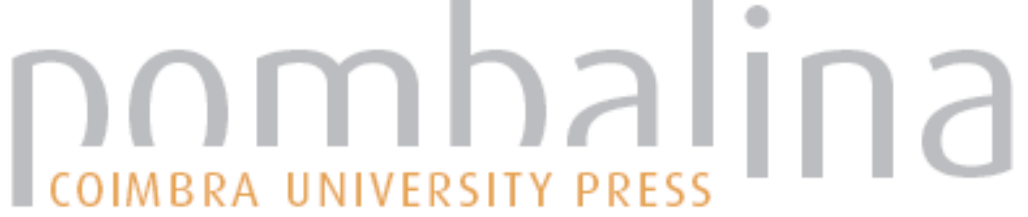

\section{A Criança submetida a Transplante de órgãos sólidos}

\author{
Autor(es): Gonçalves, Isabel
}

Publicado por: Imprensa da Universidade de Coimbra

URL

persistente: URI:http://hdl.handle.net/10316.2/43144

DOI: $\quad$ DOI:https://doi.org/10.14195/978-989-26-1300-0_43

Accessed : $\quad$ 26-Apr-2023 10:17:25

A navegação consulta e descarregamento dos títulos inseridos nas Bibliotecas Digitais UC Digitalis, UC Pombalina e UC Impactum, pressupõem a aceitação plena e sem reservas dos Termos e Condições de Uso destas Bibliotecas Digitais, disponíveis em https://digitalis.uc.pt/pt-pt/termos.

Conforme exposto nos referidos Termos e Condições de Uso, o descarregamento de títulos de acesso restrito requer uma licença válida de autorização devendo o utilizador aceder ao(s) documento(s) a partir de um endereço de IP da instituição detentora da supramencionada licença.

Ao utilizador é apenas permitido o descarregamento para uso pessoal, pelo que o emprego do(s) título(s) descarregado(s) para outro fim, designadamente comercial, carece de autorização do respetivo autor ou editor da obra.

Na medida em que todas as obras da UC Digitalis se encontram protegidas pelo Código do Direito de Autor e Direitos Conexos e demais legislação aplicável, toda a cópia, parcial ou total, deste documento, nos casos em que é legalmente admitida, deverá conter ou fazer-se acompanhar por este aviso.

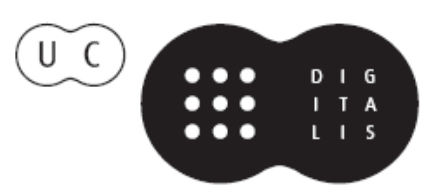




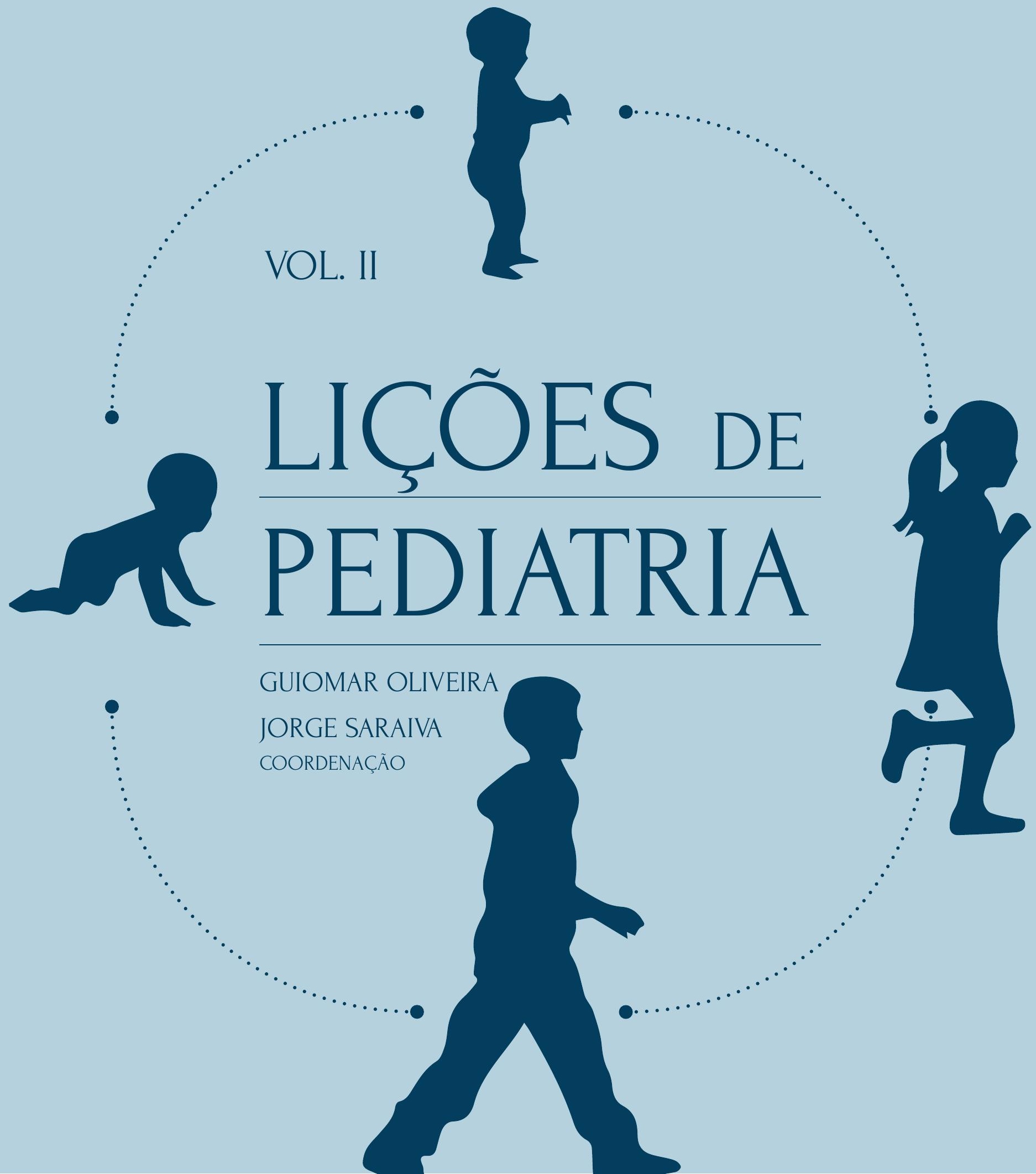


Capítulo 43.

A Criança submetida a Transplante de órgãos sólidos
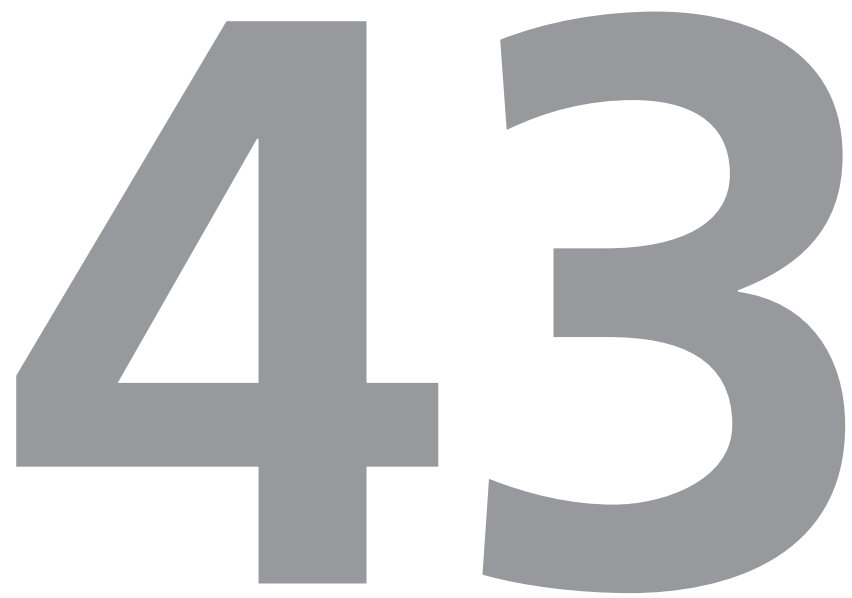

\section{Isabel Gonçalves}




\subsection{CONTEXTO}

A transplantação de órgãos sólidos em idade pediátrica iniciou-se no século $X X$, na década de 60. É uma história de sucesso e insucesso, de desafios e inovação, considerada experimental até meados dos anos 80 e desde então com um lugar aceite na Medicina contemporânea. Está longe de ser uma ciência baseada na evidência, embora protocolada e acolhendo grupos de trabalho nas várias áreas: rim, fígado, pulmão, coração e intestino. O transplante de pâncreas que se pratica em adultos não tem indicações em pediatria e por isso não será considerado neste texto.

O primeiro transplante com sucesso a longo prazo foi um transplante renal (quadro 1). Foi simultaneamente o primeiro Dador Vivo (DV), entre gémeos idênticos contornando assim o problema da rejeição numa era em que apenas os corticoides e a azatioprina estavam disponíveis como imunossupressores.

\section{4}

First successful KIDNEY transplantation (Boston, USA)

\section{7}

First successful LIVER transplant (Denver, USA)

\section{4}

First successful HEART transplant (Stanford, USA)

\section{7}

First successful LUNG transplant (Toronto, CA)

\section{9}

First successful BOWEL transplant (Paris, FR)

Quadro 1. Primeiros transplantes de órgãos sólidos bem sucedidos.
Na década de 80 surge a ciclosporina como imunossupressor principal permitindo o desenvolvimento sustentado da atividade de transplantação em todo o mundo. Atualmente o número de transplantes pediátricos, por ano na Europa estima-se em 3.000 sendo o rim o de maior expressão numérica. No registo UNOS (United Network for Organ Sharing USA) foram realizados em 2012, 1.509 transplantes de órgãos sólidos em crianças (1 aos 17 anos inclusive) com sobrevida no primeiro ano superior a 90\%. O transplante renal representa $42 \%$, fígado $28 \%$, coração 22 $\%$, intestino e pulmão cerca de $2 \%$ cada. Em Portugal em 2013 houve 17 transplantes renais, 11 de fígado e dois de coração em idade pediátrica.

\subsection{DESCRIÇÃO DO TEMA}

\subsubsection{Doação}

Os dadores para pediatria nos países ocidentais são alocados de acordo com a lei vigente em cada país (em Portugal todos os não inscritos no Registo Nacional de Não Dadores - RENNDA) sendo a maioria dos transplantes efetuada com Dador Cadáver (DC) em morte cerebral. A utilização de DC em paragem cardíaca é recente e representa menos de 5 \% na Europa e Estados Unidos. O DV foi durante muitos anos a única opção em países como o Japão, por razões religiosas. $\mathrm{Na}$ Europa a transplantação de DV representa 20 a $25 \%$ do total de transplantes hepáticos pediátricos e menos de $20 \%$ no rim. Tem vantagens em termos de sobrevida mas envolve mais recursos técnicos e financeiros e eticamente continua a ser discutível como primeira opção em países com 
taxas elevadas de doação, como por exemplo em Portugal. Muito controversa continua a ser a sua indicação no transplante emergente de fígado. Por razões óbvias é possível efetuar DV em todos os transplantes de órgão sólido à exceção do transplante cardíaco.

\subsubsection{Procedimento}

Tecnicamente todos os órgãos envolvem especificidades próprias mas em comum todos partilham três passos fundamentais: i) anastomoses arteriais; ii) anastomoses venosas, e iii) anastomoses de vias de drenagem de fluidos ou ar: biliar, ureter, intestino e traqueia, (exceção do coração que só tem anastomoses vasculares). O fígado tem o transplante mais complexo com tempos de cirurgia, de 6 a 12 horas. A maioria das crianças (80\%) recebe fígados de adultos que são geralmente reduzidos para permitir compatibilidade anatómica. Nos restantes órgãos o implante é de órgão inteiro implicando dadores anatomicamente compatíveis o que aumenta o tempo em lista de espera.

Os resultados hoje (quadro 2) devem-se a três fatores fundamentais: curva de aprendizagem técnica; microcirurgia nas anastomoses vasculares; otimização na seleção de dadores-recetores e meIhoria na imunossupressão.

\subsubsection{Indicações}

Em contraste com os adultos a maioria das patologias que leva ao transplante em crianças resulta de malformações congénitas, anatómicas ou funcionais. O momento da decisão de transplante é condicionado por critérios estabelecidos baseados ou não em fórmulas de auxílio à estratificação (i.e. Pediatric End-Stage Liver Disease - PELD). Todos os órgãos partilham como critério major a falência terminal de órgão e /ou a refratariedade às terapêuticas instituídas. Insuficiência aguda só é considerada no fígado e miocárdio e é particularmente emergente no transplante hepático. Com efeito o suporte artificial de órgãos (diálise, coração de Berlim, Extracorporeal membrane oxygenation- ECMO) permite um tempo de espera em lista alargado que pode ser indefinido no caso do rim. O sistema, Molecular Adsorbent Recycling System - MARS, ou Bioartificial Liver device - BAL, para o fígado não tem a mesma eficácia pelo que na prática são considerados "pontes" para a transplantação e não terapias substitutivas de função de órgão.

\begin{tabular}{|l|c|c|c|c|c|c|}
\cline { 2 - 7 } \multicolumn{4}{c|}{} & \multicolumn{2}{l|}{ Sobrevida pacientes (\%) } & enxertos (\%) \\
\cline { 2 - 7 } \multicolumn{1}{c|}{} & 1 ano & 5 & 10 & 1 ano & 5 & 10 \\
\hline Rim & 98 & 96 & 92 & 96.5 & $84-87$ & 54 \\
\hline Fígado & $84-90$ & $82-85$ & 77 & $84-93$ & $81-88$ & 75 \\
\hline Coração & 80 & 68 & 58 & $86-90$ & $68-75$ & - \\
\hline Pulmão & 83 & 54 & 44 & $78-88$ & $35-41$ & - \\
\hline Intestino & $80-95$ & 77 & 46 & 88 & 74 & 58 \\
\hline
\end{tabular}

Quadro 2. Sobrevida de pacientes e enxertos por tipo de transplante. 
Do ponto de vista imunológico a seleção dos recetores é organizada de acordo com a compatibilidade do sistema ABO. Em crianças pequenas (primeiro ano de vida) podem ser usados órgãos ABO - incompatíveis com resultados de sobrevida de enxertos semelhantes. No entanto esta prática deve ser reservada para casos muito selecionados.

Nos transplantes cardíacos e renais há ainda que garantir uma boa compatibilidade HLA e verificar a presença de allo-anticorpos preformados.

\subsubsection{Complicações}

Dividem-se em precoces (primeiros seis meses) e tardias (depois dos seis meses). Há complicações específicas de cada órgão transplantado mas é possível estabelecer um paralelismo para os diferentes órgãos.

Nas primeiras semanas temos complicações:

1. vasculares (tromboses dos vasos anastomosados), incidência variável nos vários transplantes (no figado afeta 2 a $10 \%$ dos doentes).

2. infeções da "ferida cirúrgica" ocorrem em $60 \%$ dos pacientes (geralmente por bactérias gram negativas, muitas delas multirresistentes).

3. não função de órgão (primary non-function, PNF) e problemas em anastomoses específicas (biliar, ureteres, traqueia) em 5 a $20 \%$ dos casos.

4. rejeição: a rejeição celular aguda (RCA) é a mais frequente. No rim e no coração a rejeição humoral (mediada por anticorpos preformados ou "de-novo" dirigidos a antigénios do dador) ainda ocasiona morbilidade. A RCA tem uma incidência de 40 a $60 \%$ nas primeiras seis semanas no transplante de fígado, 30 a $40 \%$ no rim, coração e pulmão inferior a 10\% mas a documentação sistemática por biópsia do enxerto é menor nestes órgãos.

5. são ainda comuns as reativações ou primoinfeções assintomáticas dos vírus cítomegalovirus (CMV) e Epstein-barr vírus (EBV) em relação com o grau de imunossupressão do recetor e do estado serológico pré transplante dos dadores e recetores. A infeção pelo CMV é precoce (duas a seis semanas pós cirurgia) e é particularmente nociva no transplante de rim onde foi amplamente estudada.

A medicação nos primeiros seis meses consiste em vários tipos de fármacos dos quais os imunossupressores são o pilar fundamental na profilaxia da rejeição. Os protocolos variam entre os diferentes órgãos sendo a imunossupressão geralmente tripla nos primeiros seis meses e baseada em tacrolimus ou ciclosporina (inibidores das calcineurinas), prednisolona e antiproliferativos (azatioprina ou micofenolatos). O imunossupressor principal é doseado em cada consulta e os níveis a atingir variam também entre os diferentes transplantes (menor no fígado e mais elevados no intestino, coração e rim).

Nos primeiros seis meses os doentes tomam em média seis a oito fármacos diferentes além dos imunossupressores já referidos: aspirina e dipiridamol (prevenção de trombose), aciclovir ou valganciclovir (profilaxia de Herpes, CMV e EBV), cotrimoxazol (prevenção das infeções por Nocardia spp e Pneumocystis jiroveci) e antihipertensores (seleção do fármaco varia nos diferentes órgãos transplantados). 


\section{Complicações tardias}

Com as atuais taxas de sobrevida ao fim de um ano (90\%) as crianças com transplante de órgãos são uma população crescente de regresso a casa, à escola e a uma vida com regras e cuidados pouco diferentes dos seus pares. Naturalmente que em $20 \%$ as complicações recorrentes (rejeição, infeções relacionáveis com o tipo de cirurgia) determinam várias hospitalizações e é causa de morbilidade e até mortalidade nesta fase. Mas a grande maioria está bem, embora necessite de consultas cada três ou quatro meses com avaliações analíticas e dos fármacos imunossupressores e outros exames de imagem ou histológicos (biópsias) de acordo com o protocolo de vigilância.

Em ambulatório há alguns aspetos a sublinhar e que devem fazer parte do conhecimento geral. As vacinas com vírus vivos e/ou atenuados não são recomendadas depois do transplante e a vacina antisarampo papeira e rubéola (VASPR) deve ser antecipada (seis ou nove meses idade) quando se prevê que o transplante possa ser necessário pelos 12 meses. O contacto com varicela ou outras epidemias na escola ou na comunidade devem ser notificadas ao centro de transplante e a profilaxia quando existe deve ser iniciada de imediato. A febre nem sempre é elevada ou pode estar ausente no decurso de infeções graves nas crianças imunodeprimidas. Em caso de diarreia alguns imunossupressores como o tacrolimus /everolimus e sirolimus aumentam as concentrações. Neste contexto o fármaco deve ser diminuído em $50 \%$ da dose até resolução do quadro. As interações entre os imunossupressores e alguns fármacos devem ser lembradas aquando da sua prescrição. As crianças transplantadas possuem livros de orientação/registo e contactos (telefone, email) que devem ser disponibilizados sempre que observados fora do seu Centro habitual.

Com a melhoria da sobrevida surgem novos problemas a médio e a longo prazo relacionáveis com a medicação (cardiovasculares, renais, diabetes - New Onset Diabetes After Transplant - NODAT, tumores) mas também com o estilo de vida e a capacidade educativa necessária neste contexto.

Renais e cardiovasculares:

São mais frequentes no transplante renal e cardíaco mas nos últimos anos com protocolos de vigilância mais específicos, verifica-se que por exemplo no transplante hepático e em pacientes estáveis, 15\% com mais de dez anos sobre o transplante apresenta taxas de filtração glomerular inferiores a $60 \mathrm{ml} /$ minuto/1.73 m2. A noção de lesão renal progressiva é recente e obriga a avaliação de co-morbilidades como a hipertensão arterial e a diabetes, mas sobretudo à adaptação precoce dos esquemas de profilaxia da rejeição, diminuindo as doses ou mesmo suspendendo os inibidores da calcineurina. A avaliação sistemática da tensão arterial nestes pacientes deve ser encarada como uma norma em doente de risco. O registo de alterações obriga a monotorização de 24 horas em ambulatório.

Rejeição crónica e disfunção crónica dos enxertos: um número crescente de imunossupressores mais potentes, permitindo variadas combinações não reduziu a taxa de rejeição crónica ao longo dos anos. No fígado situa-se entre 3 e 8 \% e a taxa é crescente para os restantes órgãos atingindo um valor máximo de $80 \%$ no intestino. É curioso que o fígado que tem a mais alta taxa 
de rejeição celular aguda se torne ao longo dos anos o enxerto mais tolerado (com sobrevidas superiores a $75 \%$ aos 20 anos em contraste com apenas 30 a $40 \%$ dos rins transplantados). Muito há ainda para investigar e aprender na complexa relação enxerto-hospedeiro. A rejeição crónica é uma das razões de retransplante condicionando morbilidade e mortalidade não negligenciável.

\section{Tumores de "novo":}

Surgem em crianças transplantadas, numa percentagem quatro a cinco vezes superior à esperada na mesma faixa etária. Embora os tumores malignos (pele, intestino, tiroide) surjam de forma idêntica à dos adultos, nas crianças há uma situação predominante que é a emergência de linfomas em associação com a infeção pelo vírus EBV. Estes tumores designados por, Post Transplant Lymphoproliferative disease- PTLD, envolvem geralmente as amigdalas e /ou o tecido linfoide intestinal mas podem surgir em qualquer órgão, tendo tropismo particular para os enxertos. A determinação regular das cargas virais do EBV, o diagnóstico precoce e a suspensão ou redução da imunossupressão, mantendo apenas os corticoides, permitem a involução do PTLD em 90 \% dos pacientes. Nos restantes uma estratégia combinada com quimioterapia e rituximab (anticorpo monoclonal anti CD20) é necessária, tendo este grupo uma maior morbilidade e risco de morte. Os PTLD têm muito baixa incidência no transplante de rim, moderada no fígado e pulmão e muito elevada no transplante de coração e intestino.

Os tumores transmitidos pelo dador (envolvendo o enxerto na maioria) ocorrem num número reduzido de crianças $(<0,03 \%)$ mas o diagnóstico é sempre complexo.

\section{Crescimento:}

A estatura final projetada numa criança em função dos progenitores não se aplica infelizmente na grande maioria de crianças recetoras de órgãos mas é uma preocupação real dos cuidadores destas crianças. Por um lado a estatura é um marcador de bem estar físico, de saúde, e por outro lado estabelece diferenças na aceitação pelos pares. É um problema também emergente na última década com a melhoria de sobrevida e nesse sentido vários estudos analisaram não só o perfil de crescimento das crianças mas fatores passíveis de o melhorar. Os corticoides relacionam-se negativamente com a velocidade de crescimento e assistimos a um número crescente de protocolos de imunossupressão sem corticoides "ab initium" ou que visam a sua suspensão nos primeiros três meses pós transplante. A disfunção crónica de enxerto (independente da etiologia) pela inflamação associada e a necessidade de retomar ou aumentar os corticoides representa outro importante fator sobretudo nas crianças com transplante renal e de fígado. Por outro lado tem impacto positivo a idade em que ocorre o transplante, tendo as crianças mais pequenas (menos de dois anos) maior crescimento de retorno em comparação com as mais velhas. As crianças com transplante cardíaco e sem corticoides na fase de manutenção têm velocidade de crescimento normal.

\subsubsection{Qualidade de vida/ adaptação social e escolar /adolescência}

Vencidas as dificuldades e complicações do primeiro ano após o transplante, torna-se premente delinear um suporte global de completa integração da criança com transplante de órgãos e 
respetivas famílias. A qualidade de vida (QOL) tem sido objeto de inúmeras publicações e projetos de investigação e engloba áreas diversas como a adaptação social e escolar, desenvolvimento e crescimento neurocognitivo, comportamento individual e familiar, adesão terapêutica, puberdade, contraceção e fertilidade.

De um modo geral (e para os vários tipos de transplante) as crianças e jovens apresentam maiores dificuldades escolares e taxas de insucesso (30 a $40 \%$ em várias séries) e frequentes alterações do comportamento e dificuldades na interação / integração social. A "desculpabilização " dos cuidadores (pais, tutores e mesmo técnicos de saúde) face à gravidade das doenças a que foram expostos, é muitas vezes apontada como um dos elementos com impacto negativo mais significativo. No entanto, ao rever a vastíssima literatura sobre este tema, facilmente se percebe que os aspetos neurocognitivos e comportamentais da criança com transplante de órgãos são complexos e que faltam estratégias de intervenção preventiva, individuais e familiares.

Quando avaliados por questionários sobre qualidade de vida, adaptados à sua condição as crianças e jovens obtêm índices de resposta sobreponíveis aos seus pares, em nítido contraste com a resposta dos pais ou outros cuidadores que é invariavelmente menos positiva.

A adolescência é definitivamente um período complicado no seguimento destes doentes. A maioria sente-se fisicamente bem e como qualquer adolescente vai tentar testar os seus limites. O problema mais comum e expectável é a não adesão terapêutica, outra área que tem merecido alguma atenção de grupos de investigadores. Desenvolveram mesmo alguns modelos matemáticos baseados nas variações de níveis de imunossupressores ao longo do tempo para permitir classificar e quantificar o grau de adesão ou não adesão. Clinicamente é difícil e sobretudo delicado confrontar um doente e / ou a família com a falha de cumprimento terapêutico.

A puberdade é tipicamente diferida (um a dois anos) nas crianças transplantadas mas recuperável e sem repercussões futuras se o enxerto se mantiver estável. Quando a disfunção de enxerto é grave e / ou persistente surgem alterações hormonais que em conjunto com a medicação promovem disfunções pubertárias. Nas raparigas dismenorreia e amenorreia primária ou secundária ocorrem em metade dos casos.

As adolescentes devem ser orientadas para uma consulta de ginecologia de adolescentes de forma idêntica à da população saudável. Da mesma forma os métodos usuais indicados na população saudável podem ser aplicados embora a vigilância de efeitos secundários e a interação com os fármacos necessários ao transplante obrigue a uma abordagem modificada sempre em articulação com o Centro de Transplante.

Na transição para o hospital de adultos é fundamental que o relatório operatório (no caso de transplante de órgãos abdominais) seja enviado e as jovens são advertidas que o seu obstetra deve ter conhecimento desse mesmo relatório em caso de gravidez. 


\subsection{FATOS A RETER}

As crianças com transplante de órgãos sólidos têm hoje elevada sobrevida.

Todos os clínicos irão contactar com esta nova área da medicina.

O crescimento em todas as vertentes, a morbilidade cardiovascular e renal e a emergência de tumores devem ter monitorização interdisciplinar.

A integração social e escolar, a adesão ao tratamento, a promoção de estilos de vida saudáveis exigem o seguimento desta população em consultas de Pediatria do neurodesenvolvimento já existentes nalguns Centros Hospitalares.

Imunossupressão "por medida" ou viver sem imunossupressão constitui o desafio da próxima década. 